\title{
Horses for courses: choosing an evidence-based psychological therapy for your patient
}

\author{
David McCormack \& Trudie Chalder
}

David McCormack is a clinical psychologist at the Maudsley Hospital, South London and Maudsley NHS Foundation Trust, London, UK and a lecturer and clinical tutor on the DClinPsych programme at Queen's University Belfast. Trudie Chalder is Professor of Cognitive Behavioural Psychotherapy at the Institute of Psychiatry, Psychology and Neuroscience, King's College London, and Director of the Persistent Physical Symptoms Research and Treatment Service, South London and Maudsley NHS Foundation Trust. She was President of the British

Association of Behavioural and Cognitive Psychotherapies between 2012 and 2015, and is also a practising cognitive-behavioural psychotherapist.

Correspondence Professor Trudie Chalder, Institute of Psychiatry,

Psychology and Neuroscience, King's College London, UK. Email: trudie.

chalder@kcl.ac.uk

\section{Copyright and usage}

(c) The Royal College of Psychiatrists 2018

\section{SUMMARY}

Choosing an intervention for a patient experiencing distressing symptoms and/or suffering with a mental disorder is part of routine practice for clinicians. While there are now many effective pharmacological and psychological therapies for mental health problems, syndromes and persistent physical symptoms (e.g. chronic pain), choosing the 'right' therapy can sometimes be a challenge. This can certainly be the case when it comes to psychological therapies. There are many different approaches to choose from and many have not been subjected to rigorous study.

In this article, we aim to help inform and guide the busy clinician in choosing a psychological therapy for their patient. We give a brief overview of the major psychotherapy modalities and consider which guidelines to refer to and which psychological therapies have been found to be most effective for the presenting problem(s) seen in clinic. Lastly, we discuss the limitations of the current guidelines in relation to comorbid presentations and consider how this can be best addressed.

\section{LEARNING OBJECTIVES}

- Develop knowledge regarding the major psychotherapy approaches

- Be aware that there is no psychotherapy equivalence

- Learn that there is good evidence that some approaches are more effective than others for specific problems and be better able to choose a psychological therapy

\section{DECLARATION OF INTEREST}

D. McC. works on research trials funded by the Guy's and St Thomas' Charity. T. C. receives salary support from the National Institute for Health Research (NIHR) Mental Health Biomedical Research Centre at South London and Maudsley NHS Foundation Trust and King's College London, and receives several grants for evaluating the efficacy of different cognitive-behavioural approaches. This article received no specific grant from any funding agency, or from the commercial or not-for-profit sectors. The views expressed in this article are those of the authors and not necessarily those of the NIHR or the NHS.

\section{Keywords}

Evidence; psychotherapy; formulation; psychological treatments; psychotherapy.

Pioneering work by Janet (1901) and Breuer \& Freud (1895) demonstrated that non-invasive treatment (i.e. 'talking therapy') could enable patients to overcome emotional and psychological disturbance. Following on from the work of these early pioneers there has been considerable progress in our understanding of what maintains common mental health conditions and syndromes, and how psychological therapies can help to treat such difficulties. Progress during the 20th century was made possible by advances in areas such as behavioural and cognitive psychology, refinement of psychoanalytic theory, existential philosophy, and the development of cybernetics and systems theory as applied to human psychology. As a result of this fertile period of development, we now have many forms of psychotherapy, from art therapies through to Zen therapy. Of these many and varied approaches, a smaller number have been well developed and subjected to rigorous empirical study. In this article, we limit our focus to the major modalities.

\section{The major modalities}

Broadly speaking, we can classify psychotherapy into four major modalities:

- psychoanalytic/psychodynamic

- cognitive and behavioural

- humanistic and existential

- systemic.

Each of these modalities should be considered not as representing a single therapeutic approach, but rather as a broad movement of therapies which have commonalties in their philosophical and theoretical underpinnings and/or therapeutic methods, techniques and strategies. To better understand these modalities, we will briefly summarise the approaches and then outline for the reader which guidelines to refer to when choosing an effective 
psychological therapy for their patients. We will then consider some of the challenges that can occur when using the current guidelines and consider ways to address these.

\section{The psychoanalytic/psychodynamic modality}

The psychoanalytic/psychodynamic modality is a very broad and diverse movement of theories and approaches, ranging from psychosexual and psychosocial formulations through to object relations and attachment theories. It is challenging to concisely summarise what unites these diverse approaches. What arguably is at the heart of the many sophisticated theories and nuanced accounts of the human condition and psychopathology postulated under this broad umbrella of approaches is the seemingly simple idea that early experiences affect the developing intrapsychic world and that this subsequently influences and colours the experiences and behaviours of the present self. In a real and profound sense, the past is thought to influence the present. As human development is by its nature incremental, it occurs over a lifetime, and as much of mental life is unconscious, out of our awareness, it is arguable that the past, and moreover unconscious representations derived from past experiences, influences the present in terms of both our internal world and external behaviour (Bateman 2002).

Treatment varies depending on the school of thought a therapist has been trained in and/or is working from (Gabbard 2007). In general, during the course of therapy the therapist works together with the patient to enable them to explore and become conscious of what is contributing to their emotional responses and behaviours. Therapy may involve working towards resolving unconscious conflicts. To do this the therapist uses a number of methods, which often include exploring the patient's transference (Box 1).

This process of helping the patient make sense of how they feel, respond and interact with other people the way they do is a complex one. It is all too easy for the patient to resist it, responding for example with primitive defences and/or seizing on something inconsequential to explain their feelings and behaviours. There are many reasons a patient may not want to explore and search for the unknown unconscious 'content' underlying distressing feelings and behaviours. They include painful memories, recognising difficulties in past and current relationships, and potentially confronting the 'negative' side of personality, the sum of all those qualities we like to hide, that we are afraid of: the hated or feared parts of ourselves (Klein 1984).

An important aspect of modern psychoanalytic/ psychodynamic therapy is that it aims to facilitate

an empathic, containing therapeutic relationship (Bion 1962), where attachment is considered not only in terms of the patient's past, but also how it plays out in the consulting room (Ma 2006). Therapy provides an environment in which the person can make sense of why they react the way they do, process distressing memories and experiences, and better understand themselves and their relationship to others. This may lead to an improved integration of the self, where the person is less distressed and/or is better able to understand and tolerate their feelings.

\section{The cognitive and behavioural modality}

To understand the modern cognitive and behavioural approach it is useful to know a little about its roots. It is important first to note that the cognitive and behavioural modality encompasses a broad umbrella of theories and therapies, it is not one discrete approach. In fact, before the development of the cognitive approach there was simply behavioural therapy. Behavioural therapy has its origins in experimental psychology and physiology. Building on the findings of Pavlov (1927) and Watson \& Rayner (1920), experimental research quickly led to clinical application. Mowrer (1947), drawing on classical and operant conditioning theory, developed empirical formulations that cemented the foundations of behavioural therapy. For example, his work led to an elegant theory of why fear persists and does not simply fade and extinguish. Within this framework, it is argued that when people develop a fear they seek to avoid experiencing the unpleasant feeling and, through this avoidance, the conditioned response (i.e. fear) does not naturally extinguish and is maintained. Wolpe (1968) proposed using systematic desensitisation to a hierarchy of feared situations to enable people to overcome their anxiety and fears through exposure to them. That is, through exposure to the feared stimuli over time, the fear/anxiety fades (i.e. the conditioned response extinguishes). After further

BOX 1 Transference and countertransference (psychoanalytic/psychodynamic psychotherapy)

Transference is an intrapsychic phenomenon in which the patient transfers to the therapist, or someone important in their present life, the 'conflict laden ideas and feelings that are more appropriate to someone important in the person's past' (Patton 1992: p. 85). The therapist may use transference, as well as countertransference (i.e. feelings evoked in the therapist), as a way to gain insight into the patient's internal world and to uncover how the patient relates to significant others in their life. By sensitively interpreting for the patient the transference that is taking place, the therapist may give the patient an opportunity to become aware of their unconscious internal world and patterns of relating to others (Freud 1910). 


\section{BOX 2 Formulation}

Formulation can aid in the understanding of a patient's difficulties, identify what may be contributing to, exacerbating and/or maintaining problems, and help prioritise and select where to intervene (Butler 1998). As Mace \& Binyon (2005) point out, formulation can be carried out before commencing psychotherapy. It is a skill not only for clinical psychologists and psychotherapists, but a key clinical skill for all psychiatrists. There are many different ways to formulate (see e.g. Johnstone 2006a). We would recommend that formulation be developed collaboratively with the patient and be what guides and informs intervention. refinements, behavioural therapy has been successfully applied to treat a range of clinical problems.

As behavioural therapy began to be used more widely in clinical practice, it became apparent to many that a strictly behavioural approach was missing an important component, namely, it was overlooking the role of mental processes. Work by figures such as Ellis (1962) and Beck (1976, 1993) emphasised that cognitive processes play an important role in the development and maintenance of emotional disorders, and that therapy focused on cognitive factors, for instance challenging unhelpful thinking (e.g. excessively negative thoughts, cognitive distortions), could bring about behavioural change and symptom improvement. The adoption of the cognitive approach provided a dynamism that had been lacking before. This can be expressed simply in the following circular process - cognitive change influences behaviour, behaviour influences cognition, and they both affect emotions and physiology, which in turn influence cognition and behaviour. The combination of both cognitive and behavioural components has enabled the development of sophisticated formulations and treatments for a wide range of mental disorders, unexplained and persistent physical symptoms, and long-term conditions.

At the heart of the cognitive-behavioural approach is an empirical focus. The experimental roots of this approach carry through into the consulting room. This occurs by routinely subjecting the approach to rigorous monitoring and scrutiny, even at the level of individual therapy. For example, complex idiosyncratic formulations (i.e. a theoretical model of the patient's problems) are developed collaboratively with the patient and draw on, in large part, findings from empirical research. This shared formulation seeks to make sense of the patient's difficulties and will guide where and how to intervene. A cognitive-behavioural formulation is designed to be testable. That is, it enables a clear framework for the patient to test whether the intervention, the cognitive and behavioural strategies and techniques, is effective in overcoming the presenting problem(s) and in achieving treatment goals (e.g. improvement in symptoms, reducing associated distress, increasing functioning).

\section{Humanistic and existential approaches}

Humanistic and existential approaches are another broad grouping of therapies. The roots here can be traced back to continental philosophy. Humanistic therapy assumes quite an optimistic view of the human condition, one where people are naturally striving towards growth, fulfilment and actualisation; therapy within this approach aims to remove barriers to this growth and enable the person to move towards self-actualisation. An underlying assumption of this approach is that human beings have an inbuilt propensity towards self-realisation (Horney 1999) and that with the right environment, the right conditions, the person can surmount their difficulties and grow and develop as an individual. Therapy within this approach seeks to provide these conditions. That is, being accurately empathised with, listened to with unconditional positive regard by a therapist who is being genuine and congruent, provides the patient with the necessary conditions to discover for themselves how to overcome their difficulties and to develop (Rogers 1951). Although the conditions for therapy that Rogers describes within this approach are probably important to foster a good therapeutic relationship, that these conditions are sufficient for effective therapeutic change is not as well supported.

Existential approaches, in contrast to the humanistic approach, are often seen as being more firmly rooted in weighty philosophical origins and more concerned with ontological and ontic inquiry, a search for meaning, and take a more nuanced view of the human condition. While there is indeed some truth to this, particularly with regard to the British school (Spinelli 2014), the focus on supporting people to non-judgementally examine the view they hold of themselves, of life, their relationship to others, and the narratives and meanings they construct, has many parallels and similarities with humanistic approaches, perhaps not surprising given their shared philosophical roots.

Both humanistic and existential therapies place an emphasis on meaning and subjective experience, and seek to explore with the patient their unique experience of being-in-the-world. Therapy from these approaches aims, among other things, to support people to take responsibility for making their own choices and choosing how they want to act. These are approaches not fixated on strategies and techniques, which are a common feature of approaches such as cognitive-behavioural therapy. Although these are insight-oriented therapies, in 
contrast to psychoanalytic approaches, interpretation is de-emphasised whereas subjective exploration is arguably given even greater emphasis. It is this process of explorative enquiry that leads to fresh insight, and with it the freedom for the person to choose how they will respond and act in light of the insight gained.

\section{Systemic approaches}

Although the psychotherapy approaches introduced above have many differences, some obvious and others more nuanced, what they share is a focus on the individual. They often seek to understand the person's symptoms and problems as arising from within the individual, for example as a result of psychological processes and/or because of unhelpful behaviours, and interventions tend to focus on working with the individual patient to address their difficulties. Systemic approaches can be seen to stand in contrast to these other approaches, and some consider them to be radically different (Stratton 2011). Rather than focusing solely on the individual, systemic approaches take a wider focus. From a systems perspective, the individual is viewed to be affected by the setting and contexts, the systems, they find themselves in. Each individual is seen as affected by their relationships and interaction with others in the systems they are in, and they also affect the other individuals in these systems.

Some theorists have even postulated that the symptoms that an individual experiences may arise from how other people interact with them and/or that certain types of system, specifically certain families, are associated with certain disorders, for example 'psychosomatic families' (Minuchin 1978). However, there is little to no empirical evidence to support this. Most modern systemic theory and family therapy typically allows for the possibility that the original problem may have arisen for any number of reasons, but it can be maintained or exacerbated by how those in the system interact with one another, and these interactions are assumed to be driven by the range of beliefs and narratives held by those in the system.

Systemic intervention is most often carried out with families and couples, but it has even been adapted to work with individuals by supporting the individual to become more aware of the wider social context and their relationships with others in various family and social systems (Jenkins 1992). There are many forms of systemic and family therapy, but a key feature is helping individuals in the system to better understand how they relate to one another and encouraging them to deemphasise the focus on pathology and/or blaming one particular person (i.e. the identified patient). The therapist then draws on this understanding to enable those in the system to improve their way of relating to one another and, as ways of relating improve, it is assumed that this will address the presenting problem (e.g. in the case of disturbing symptoms experienced by a member of a family, these should fade or become less problematic) (Carr 2008).

\section{Similarities between the major modalities}

Although it is clear that there are many differences across the major modalities, there are similarities too. For instance, it is now widely accepted that an individual's experience, from the earliest moments onwards, affects how they think and act, relate to others and are treated by others. Attachment theory has influenced all of the major schools of therapy. Even if it is not always explicitly referenced, aspects of this theory are present in many modern formulations that seek to understand the patient's past relationships and their current ones, including the relationship in the consulting room (Ma 2006). There is also a growing appreciation of the role of unconscious processes across the psychotherapy modalities, although here considerable diversity exists regarding the conceptualisation of the unconscious and its role in therapy (e.g. Power 1991; Flaskas 2005).

Systemic elements are either implicitly or explicitly acknowledged and incorporated into theory across the modalities. That is, even in the therapies that predominantly focus on working at the individual level, an understanding of how the person relates to the systems they are in and how these systems affect the person is typically considered and incorporated into therapy (i.e. there is commonly an appreciation of the influence of systems such as family, work or school on the individual). There have even been attempts at integrating systemic elements more formally into other approaches (e.g. see Dummett 2005).

\section{Distinct and stand-alone therapies}

A number of distinct and effective therapies have developed under the broad umbrella of the major modalities. For instance, stemming from psychoanalytic/psychodynamic roots is brief psychodynamic therapy (Leichsenring 2004), and from cognitive and behavioural roots we have dialectical behaviour therapy, acceptance and commitment therapy, and mindfulness-based cognitive therapy (Leichsenring 2004; Oei 2008; Hacker 2016). Then there are therapies that draw on at least two of the major modalities, such as cognitive analytic therapy, interpersonal therapy, schema-focused therapy and 
mentalisation-based therapy (Ali 2016; Cristea 2017a; Taylor 2017). There are also therapies that developed outside of the major modalities, for example eye-movement desensitisation and reprocessing therapy, art therapies, as well as mindfulnessbased approaches unrelated to cognitive-behavioural therapy, the last of these, the mindfulness-based approaches, have equivocal support, but recent data suggest that they could be promising evidencebased treatments (Goldberg 2018). Carr (2008) and Roth \& Fonaghy (2005) provide a good summary and review of many forms of psychological therapy.

\section{Modes of delivery}

Whether the therapy is carried out with individuals, couples, families, groups or even in therapeutic communities (Pearce 2017), it often has its roots in one of the major modalities, whether it be cognitivebehavioural (e.g. behavioural couples therapy, cognitive-behavioural approaches to family therapy), psychoanalytic/psychodynamic (e.g. group analytic psychotherapy) or systemic (e.g. systemic family therapy). As regards groups, the group can be where the intervention takes place, for example a psychoeducational group where people can learn about a specific presenting problem, talk about their difficulties and develop ways to better cope, or being part of the group can itself be the therapy.

When it comes to deciding on whether a patient should be referred for individual, couples, family or group therapy, the clinician should be guided by the assessment and formulation (i.e. which format is most suitable for the patient and their presenting problem), and the evidence regarding effectiveness and guidelines (we will discuss this later in this article). When deciding on which format to refer a patient to, it is crucial that the patient's preference is always taken into consideration and, wherever, possible followed.

\section{Research evidence - what works and for whom}

There is evidence that most of the major psychotherapy approaches are effective for people presenting with a range of psychological problems (Roth 2005; Carr 2008). Evidence from randomised controlled trials and meta-analyses provide support for the effectiveness of the systemic approach (Shadish 2003), humanistic therapy (Elliot 2013), existential therapy (Vos 2015), psychodynamic therapy (Leichsenring 2004) and cognitive-behavioural therapy (Butler 2006).

However, while there is evidence that most of the major psychotherapy approaches are effective, this does not mean that we can reach the 'Dodo bird verdict', as not all psychotherapies are equivalent when it comes to outcomes. Some approaches are more effective than others at treating certain presenting problems. Additionally, given the nature of the scientific method, the evidence to support a form of psychotherapy is not static, but changeable in light of accumulating data and how data are selected and interpreted. A good example of this concerns cognitive-behavioural therapy. Johnsen \& Friborg (2015) presented a meta-analysis suggesting that cognitive-behavioural therapy was becoming less effective, but Cristea et al (2017b), using a different and arguably more rigorous methodology (e.g. they included only randomised controlled trials), argued that this was not the case.

At present, there is compelling evidence that, for certain problems, some therapies are more effective than others. In the UK, the National Institute for Health and Care Excellence (NICE) and the Scottish Intercollegiate Guidelines Network (SIGN) have carried out rigorous systematic reviews of the scientific literature and developed evidence-based guidelines on what therapies appear to be most effective for presenting problems commonly seen in clinic. We would recommend that clinicians, particularly those working in the UK, be familiar with this guidance and consult these guidelines to determine which therapy to use depending on the presenting problem. NICE guidance has a wider breadth than SIGN, and provides good guidelines for many common presentations seen in clinic, including generalised anxiety disorder, depression, social anxiety disorder, obsessive-compulsive disorder, post-traumatic stress disorder, eating disorders, personality disorders, psychosis/schizophrenia, and chronic fatigue syndrome. Some examples of the guidance are given below.

\section{NICE treatment guidelines for some common disorders}

NICE (2009a) guidelines on treating depression in adults recommend several evidence-based psychological therapies. For mild to moderate depression, initially it is recommended that 'low-intensity' interventions be trialled; these include cognitive and behavioural guided self-help and computerised cognitive-behavioural therapy. If this is not effective, or if the patient has moderate to severe symptoms, then a 'high-intensity' intervention should be considered, such as therapist-delivered cognitive-behavioural therapy, interpersonal therapy, or behavioural couples therapy for those whose relationship may be contributing to or maintaining depression. For patients with mild to moderate depression who decline these therapies, NICE recommends that short-term psychodynamic psychotherapy be considered. 
Adults considered to be at an increased risk of developing psychosis should be offered cognitivebehavioural therapy with or without family intervention; for adults experiencing psychosis, cognitive-behavioural therapy, family intervention and art therapies are recommended (NICE 2014).

There is encouraging evidence for dialectical behaviour therapy and mentalisation-based therapy for borderline personality disorder (NICE 2009b; Ali 2016).

For post-traumatic stress disorder, cognitivebehavioural therapy and eye-movement desensitisation and reprocessing therapy are recommended (NICE 2005a).

For social anxiety disorder, individual cognitivebehavioural therapy is recommended (specifically the Clark \& Wells model or Heimberg model for adults) and for those who decline this, short-term psychodynamic psychotherapy that has been developed to treat social anxiety disorder should be considered (NICE 2013).

For obsessive-compulsive disorder, cognitivebehavioural therapy (including exposure and response prevention) is recommended (NICE 2005b). Cognitive-behavioural therapy is also recommended for chronic fatigue syndrome (NICE 2007).

This is just a sample of the guidelines, and what is evident is that there are certain therapies that, according to current evidence, are more effective for some presentations than others. Keeping up to date with the current guidance is important. For presenting problems not addressed by the guidelines, the Cochrane Library (particularly the Cochrane Database of Systematic Reviews), is an excellent resource to consult.

\section{Considering complexity: the shortcomings of guidelines and how to address them}

A number of important questions arise when using NICE and related guidance in clinical practice. We will consider here the three that are most often asked of us when discussing the topic. The first is, regardless of the presenting problem, are there certain people who are more (or less) likely to benefit from psychological therapy? The second question is, what should one do when there is comorbidity, given that the guidelines are disorder specific? And related to this, third, is it not best in complex cases to integrate therapies and/or tackle transdiagnostic processes (i.e. take a symptom- or problem-based approach) to improve outcomes?

Are there certain people who are more (or less) likely to benefit from psychological therapy?

Although some research has been carried out in this area (e.g. Blenkiron 1999), there is at present insufficient evidence to make any firm recommendations. Therefore, it is important to consider and offer psychological therapy to all patients where evidencebased approaches exist to address the problems they are experiencing. However, it is of course imperative to adapt therapy to ensure that it is age-appropriate and that it is tailored to the patient's intellectual ability and that sufficient support and motivation are provided to enhance engagement.

What should one do when there is comorbidity, given that the guidelines are disorder specific?

First, it should be acknowledged that the disorderspecific nature of the guidelines is a significant shortcoming when considering a therapy for someone with comorbid presentations. It invariably presents challenges, given that the clinical reality is that comorbid and complex presentations are commonly seen in clinic.

There has been growing unease in recent years about the validity and usefulness of diagnostic categories on which the guidelines are based. Although we think it would be foolish to abandon the current diagnostic system because of its shortcomings, there does appear to be a need to modify it, and perhaps a move towards a dimensional approach would be advisable. DSM-5 (American Psychiatric Association 2013), despite its many flaws, has made efforts to incorporate the dimensional approach to diagnosis and classification, although these efforts have not been without criticism (Adam 2013).

The clinical reality is that comorbidity and complexity is the norm and not the exception. Many clinicians reading this article will regularly see patients with multiple diagnoses. However, perhaps what we are looking at is not always truly multiple disorders, but rather multiple symptoms that have a common aetiology and/or are being maintained by common processes: presentations that the current diagnostic system and guidelines do not always adequately address.

Nevertheless, even when there is complexity and comorbidity it is often possible to identify specific problems for which guidelines such as NICE and SIGN can be applied and evidence-based therapies trialled.

\section{Is it not best in complex cases to integrate therapies and/or tackle transdiagnostic processes?}

For some clinicians, their role when choosing a psychological therapy for their patient is limited to assessing, diagnosing, developing a formulation and then referring the patient to appropriate specialist services for treatment. In such circumstances, it 
will probably be sufficient to be guided by NICE and SIGN guidelines in deciding to which local or national psychotherapy service to refer the patient. As we have highlighted, the guidelines can be found to be lacking when a patient presents with complexity and comorbid conditions, in which case referring to the Cochrane Library (again, in particular, the Cochrane Database of Systematic Reviews) might be of help, as might discussing the case with colleagues who have expertise in psychological therapy.

For those delivering psychological therapy, we would argue that it is important when assessing and formulating to see whether it is possible to identify specific problems for which NICE guidance could be followed. Therefore, if during the process of assessment and formulation it becomes apparent that there are specific well-defined problems present (e.g. the patient is experiencing persistent panic attacks and depression as part of a complex trauma presentation), then serious consideration should be given to addressing the problems for which guidance exists and following protocols recommended by guidelines (e.g. NICE and SIGN) and high-quality reviews. In such a situation, addressing the most distressing or disabling problem first may prove effective. This approach is echoed in the NICE guidelines. For example, in the NICE (2009a) guidelines for treating depression in adults it is recommended that, if a patient presents with depression and symptoms of anxiety, then the first priority should usually be to treat the depression. However, it is acknowledged that it might be more appropriate to treat the anxiety difficulties first, and that this will probably improve depressive symptoms. When it comes to complex presentations for which there is no clear guidance, for example a patient with behavioural problems and disparate symptoms that do not fit diagnostic categories, then taking a formulation-driven and transdiagnostic approach may prove beneficial.

BOX 3 Some frameworks for the integration of psychotherapeutic approaches

- Theoretical integration (integrating at the theory level)

- Technical eclecticism (applying approaches that seem likely to be effective regardless of the theoretical underpinnings)

- Assimilative integration (having expertise in one form of psychotherapy and using this as a foundation while pragmatically drawing on and incorporating aspects from
Transdiagnostic approach and psychotherapy integration

Taking a transdiagnostic approach (i.e. a symptomor problem-based approach) may prove effective, particularly for complex presentations. A transdiagnostic approach involves examining for common factors that appear to contribute to and/or maintain symptoms and developing a formulation (Box 2) of presenting difficulties to guide on where to intervene. Intervention can then address the cognitive, behavioural, interpersonal and social factors that appear to be exacerbating and/or maintaining symptoms (e.g. Barlow 2010).

If assessment and formulation suggest there is a good rationale for considering integrating a number of therapeutic approaches, there are several considerations to keep in mind when embarking on this. When one considers the psychotherapy approaches presented in this article, it should be apparent that a complete integration between any or all of them would be a challenge. Epistemological incompatibilities abound, and distinct differences exist regarding how presenting problems are conceptualised and how treatment is carried out. In spite of these challenges, efforts have been made to combine and integrate approaches. This is in part because all psychotherapy approaches have both strengths and limitations and no single approach is suitable for all patients. Additionally, as mentioned above, while there are many differences between therapies, there are also similarities. That is, common factors do exist across psychotherapies.

A number of frameworks have been advanced to aid integration of psychotherapies (Box 3). However, before a clinician attempts an integrative approach, it is important that they have the sufficient knowledge, skills and competence in at least two psychotherapeutic approaches. Additionally, they should have developed a detailed formulation and have a clear rationale for using an integrative approach. University College London's online competence frameworks available at www.ucl.ac.uk is a useful resource to consult.

\section{Conclusions}

Choosing the most suitable psychological therapy for a patient can sometimes be a challenge for clinicians. Recent advancements in the rigour of assessing which psychological therapies are most effective for specific conditions has made this decision-making easier. Certain psychological therapies have been found to be more effective than others. Consulting relevant guidelines and highquality reviews, such as those from NICE, SIGN and the Cochrane Library (in particular the 
Cochrane Database of Systematic Reviews), is recommended before commencing treatment or referring a patient for psychological therapy. This should ensure that the patient will be provided with the most effective evidence-based therapy.

If specific well-defined problems are present (e.g. panic disorder, obsessive-compulsive disorder) then consideration should be given to addressing these following protocols recommended by relevant guidelines and high-quality reviews.

With complex presentations, particularly featuring comorbidity, it may prove effective to take a parsimonious approach and treat the most distressing and/or disabling problem first (following treatment protocols), and then progressively address the other presenting problems. However, identifying underlying factors/processes and addressing these might prove to be more effective. What is crucial is the use of formulation. Formulation is important in all clinical work, but when any complexity is present it is vital, as it should be what guides and informs intervention (Johnstone 2006b). Intervention should be evidenced-based where possible and appropriate to addressing the presenting problem. Where indicated, taking a transdiagnostic approach (i.e. examining for symptoms or common factors that appear to contribute to and/or maintain symptoms) and/or integrating therapeutic approaches may prove effective, but caution and careful consideration are advised here for the novice therapist. For complex presentations, it would be best that only an experienced specialist in psychological therapy should treat the patient. If a less experienced therapist undertakes such work, close clinical supervision from an experienced therapist is recommended.

When choosing a psychological therapy, in addition to assessment, formulation and consulting guidelines, it is also important to consider patient preference, for example the patient may prefer not to accept a specific therapy or prefer individual rather than group therapy. It is equally important that, in the absence of compelling evidence, patients are not excluded from being offered psychological therapy on the basis of their history, background or diagnosis. Whatever therapy is chosen, the routine monitoring of clinically relevant outcomes is recommended.

\section{References}

Adam D (2013) On the spectrum. Nature, 496: 416-8.

Ali S, Findlay C (2016) A review of NICE guidelines on the management of borderline personality disorder. British Journal of Medical Practitioners, 9(1): a909.

American Psychiatric Association (2013) Diagnostic and Statistical Manual of Mental Disorders (5th edn) (DSM-5). American Psychiatric Publishing.
Barlow DH, Farchione TJ, Fairholme CP, et al (2010) Unified Protocol for Transdiagnostic Treatment of Emotional Disorders. Oxford University Press.

Bateman A, Holmes J (1995) Introduction to Psychoanalysis: Contemporary Theory and Practice. Routledge.

Beck AT (1976) Cognitive Therapy and the Emotional Disorders. Meridian. Beck AT (1993) Cognitive therapy: nature and relation to behavior therapy. Journal of Psychotherapy Practice and Research, 2: 342-56.

Bion WR (1962) Learning from Experience. Heinemann.

Blenkiron $P$ (1999) Who is suitable for cognitive behavioural therapy? Journal of the Royal Society of Medicine, 92: 222-9.

Breuer J, Freud S (1895) Studies on hysteria. Reprinted (1953-1974) in the Standard Edition of the Complete Psychological Works of Sigmund Freud (trans \& ed J Strachey), vol. 2. Hogarth Press.

Butler G (1998) Clinical formulation. In Comprehensive Clinical Psychology. Volume 6 Adults: Clinical Formulation \& Treatment (eds AS Bellack, M Hersen): 1-24. Pergamon.

Butler AC, Chapman JE, Forman EM, et al (2006) The empirical status of cognitive-behavioral therapy: a review of meta-analyses. Clincal Psychology Review, 26: 17-31.

Carr A (2008) What Works with Children, Adolescents, and Adults? A Review of Research on the Effectiveness of Psychotherapy. Routledge.

Cristea IA, Gentili C, Cotet C (2017a) Efficacy of psychotherapies for borderline personality disorder: a systematic review and meta-analysis. JAMA Psychiatry, 74: 319-28.

Cristea IA, Stefan S, Karyotaki K, et al (2017b) The effects of cognitive behavioral therapy are not systematically falling. Psychological Bulletin, 143(3): 326-40

Dummett N (2005) Processes for systemic cognitive-behavioural therapy with children, young people and families. Behavioural and Cognitive Psychotherapy, 34: 179-89.

Elliott R, Watson J, Greenberg LS, et al (2013) Research on humanisticexperiential psychotherapies. In Bergin \& Garfield's Handbook of Psychotherapy and Behavior Change (ed MJ Lambert): 495-538. Wiley.

Ellis A (1962) Reason and Emotion in Psychotherapy. Citadel Press.

Flaskas C (2005) Psychoanalytic ideas and systemic family therapy: revisiting the question 'why bother?'. Australian and New Zealand Journal of Family Therapy, 26: 125-34.

Freud S (1910) The origin and development of psychoanalysis. American Journal of Psychology, 21: 181-96.

Gabbard GO (2007) Major modalities: psychoanalytic/psychodynamic. In Oxford Textbook of Psychotherapy (eds GO Gabbard, JS Beck, J Holmes): 3-13. Oxford University Press.

Goldberg SB, Tucker RP, Greene PA, et al (2018) Mindfulness-based interventions for psychiatric disorders: a systematic review and meta-analysis. Clinical Psychology Review, 59: 52-60.

Hacker T, Stone P, MacBeth A (2016) Acceptance and commitment therapy - Do we know enough? Cumulative and sequential meta-analyses of randomized controlled trials. Journal of Affective Disorders, 190: 551-65.

Horney K (1999) Neurosis and Human Growth. Routledge.

Janet $\mathrm{P}$ (1901) The Mental State of Hystericals. Putnam's Sons.

Jenkins H, Asen K (1992) Family therapy without the family: a framework for systemic practice. Journal of Family Therapy, 14: 1-14.

Johnsen TJ, Friborg 0 (2015) The effects of cognitive behavioral therapy as an anti-depressive treatment is falling: a meta-analysis. Psychological Bulletin, 141: 747-68.

Johnstone L, Dallos R (2006a) Formulation in Psychology and Psychotherapy: Making Sense of People's Problems. Routledge.

Johnstone L (2006b) Controversies and debates about formulation. In Formulation in Psychology and Psychotherapy: Making Sense of People's Problems (eds L Johnstone, R Dallos): 208-31. Routledge.

Klein M (1984) Envy and Gratitude and Other Works 1946-1963. Hogarth Press.
MCO answers

$1 d \quad 2$ c 3 a 4 e $5 d$ 
Lampropoulos GK (2001) Bridging technical eclecticism and theoretical integration: assimilative integration. Journal of Psychotherapy Integration, 11: 5-19.

Leichsenring F, Rabung S, Leibing E (2004) The efficacy of short-term psychodynamic psychotherapy in specific psychiatric disorders: a meta-analysis. Archives of General Psychiatry, 61: 1208-16.

Ma K (2006) Attachment theory in adult psychiatry. Part 1: conceptualisations, measurement and clinical research findings. Advances in Psychiatric Treatment, 12: 440-9.

Mace C, Binyon S (2005) Teaching psychodynamic formulation to psychiatric trainees. Part 1: basics of formulation. Advances in Psychiatric Treatment, 11: 416-23.

Minuchin S, Rosman BL, Baker L (1978) Psychosomatic Families. Harvard University Press.

Mowrer OH (1947) On the dual nature of learning - a re-interpretation of 'conditioning' and 'problem-solving'. Harvard Educational Review, 17: $102-48$

NICE (2005a) Post-traumatic Stress Disorder (PTSD): Management (NICE Clinical Guideline CG26)

NICE (2005b) Obsessive-Compulsive Disorder: Treatment (NICE Clinical Guideline CG31).

NICE (2007) Chronic Fatigue Syndrome/Myalgic Encephalomyelitis lo Encephalopathy): Diagnosis and Management (NICE Clinical Guideline CG53).

NICE (2009a) Depression in Adults: Recognition and Management (NICE Clinical Guideline CG90)

NICE (2009b) Borderline Personality Disorder: Recognition and Management (NICE Clinical Guideline CG78).

NICE (2013) Social Anxiety Disorder: Recognition, Assessment and Treatment (NICE Clinical Guideline CG159).

NICE (2014) Psychosis and Schizophrenia in Adults: Prevention and Management (NICE Clinical Guideline CG178).

Norcross JC, Goldfried MR (2005) Handbook of Psychotherapy Integration. Oxford University Press.
Oei TPS, Dingle G (2008) The effectiveness of group cognitive behaviour therapy for unipolar depressive disorders. Journal of Affective Disorders, 107: 5-21

Patton MJ, Meara NM, Robbins SB (1992) Psychoanalytic Counseling. John Wiley \& Sons

Pavlov IP (1927) Conditioned Reflexes. Oxford University Press.

Pearce S, Scott L, Attwood G, et al (2017) Democratic therapeutic community treatment for personality disorder: randomised controlled trial. British Journal of Psychiatry, 210: 149-56.

Power M, Brewin CR (1991) From Freud to cognitive science: a contemporary account of the unconscious. British Journal of Clinical Psychology, 30: 289-310.

Rogers C (1951) Client-Centered Therapy. Constable.

Roth A, Fonaghy P (2005) What Works for Whom? A Critical Review of Psychotherapy Research. Guilford Press.

Shadish WR, Baldwin SA (2003) Meta-analysis of MFT interventions. Journal of Marital and Family Therapy, 29: 547-70.

Spinelli E (2014) Practising Existential Therapy. Sage.

Stratton P (2011) The Evidence Base of Systemic Family and Couples Therapies. Association for Family Therapy.

Taylor CDJ, Bee P, Haddock G (2017) Does schema therapy change schemas and symptoms? A systematic review across mental health disorders. Psychology and Psychotherapy: Theory, Research and Practice, 90: 456-79.

Tarrier N, Johnson J (2015) Case Formulation in Cognitive Behaviour Therapy: The Treatment of Challenging and Complex Cases (2nd edn). Routledge.

Vos J, Craig M, Cooper M (2015) Existential therapies: a meta-analysis of their effects on psychological outcomes. Journal of Consulting and Clinical Psychology, 83: 115-28.

Watson JB, Rayner R (1920) Conditioned emotional reactions. Journal of Experimental Psychology, 3: 1-14.

Wolpe J (1968) Psychotherapy by reciprocal inhibition. Conditional Reflex, 3: $234-40$ 
MCOs

Select the single best option for each question stem

1 As regards the statement that all psychological therapies are equally effective for treating obsessive-compulsive disorder:

a yes, that is true

b the evidence is that existential therapy works best

c NICE recommends a systemic approach as the first-line psychological therapy

d cognitive-behavioural therapy is the most effective psychological therapy for that disorder

e cognitive-behavioural therapy is most effective for that disorder when combined with psychoanalytic therapy.

2 As regards the statement that the therapeutic relationship is not that important for psychotherapy to be effective:

a that is true, it is not that important in modern psychological therapies

b it is only a concern for psychoanalytic/psychodynamic and humanistic approaches

c it is crucial to pay attention to and foster the therapeutic relationship, as it is central to effective psychological therapy

d it is only important to consider whether the patient is not engaging well in therapy

e it is of little importance in cognitive-behavioural therapy.
3 In complex cases, a transdiagnostic approach:

a is likely to be particularly suitable when comorbidity is present

b should only be used when there is no evidence of comorbidity

c should never be used

d can be used for anxiety disorders but not depression

e is only suitable for emotional problems and not persistent and unexplained physical symptoms.

4 As regards the statement that short-term psychodynamic therapy is the most effective therapy for treating social anxiety disorder:

a yes, that is true

b the evidence is that short-term psychodynamic therapy is not at all effective for that disorder

c NICE recommends existential therapy as the most clinically effective and cost-effective psychological therapy for that disorder

$d$ it is not the most effective, but is more effective than the Clark and Wells model of cognitive therapy

e it is effective, but cognitive-behavioural therapy is more effective, particularly the Clark and Wells model or Heimberg model.
5 As regards the statement that systemic approaches should never be integrated with other approaches:

a that is well supported by the available evidence

b no, systemic approaches may be integrated with humanistic and existential approaches as they are epistemologically compatible

c no, systemic approaches may be integrated with psychoanalytic/psychodynamic approaches as they are epistemologically compatible

d most major therapeutic modalities incorporate systemic elements implicitly or explicitly, and there have even been attempts to formally incorporate them with other approaches

e elements can be integrated with all approaches except cognitive-behavioural therapy. 